GLIA

\title{
Essential assemblies
}

Nodes of Ranvier are important for rapid, saltatory nerve conduction. Although the molecular constituents of the nodes and paranodes are well established, how they are assembled is still a mystery. Now, a study shows that two isoforms of neurofascin, Nfasc155 and Nfasc186, have distinct and crucial roles in the formation of functional nodes of Ranvier.

The nodes contain clusters of protein complexes, which consist of sodium channels, Nfasc186 and neuronal-glial-related cell adhesion molecule (NRCAM), as well as structural proteins such as $\beta I V$-spectrin and ankyrin G. At the paranodal axonal-glial junctions on either side of the node, Nfasc155, which is expressed by myelin-forming glial cells, binds to CASPR (contactin- associated protein) and contactin on the axon.

In this study, Sherman and colleagues found that in mice lacking neurofascin, the myelin sheath is grossly normal, but the velocities of nerve conduction are remarkably reduced compared with those of control animals. Close examination of myelin ultrastructure in these mice show that CASPR and contactin are absent from the paranodal junctions, and that sodium channels, NRCAM, $\beta I V$-spectrin and ankyrin $G$ are no longer concentrated at the nodes. As the expression and localization of these molecules are normal in mutant mice, these data indicate that neurofascin is crucial for proper assembly of nodal and paranodal complexes. Transgenic expression of Nfasc155 in the myelinating glia of neurofascin-deficient mice rescues the axonal-glial adhesion complex by recruiting CASPR and contactin, but has no effect on the assembly of nodal complexes.

The distinct functions of Nfasc155 and Nfasc186 in nodal and paranodal assemblies are intriguing. Differential expression of neurofascin in glia and neurons might have a fundamental role in the evolution of saltatory nerve conduction in vertebrates.

Jane Qiu

ORIGINAL RESEARCH PAPER Sherman, D. et al Neurofascins are required to establish axonal domains or saltatory conduction. Neuron $\mathbf{4 8 , 6 4 5 -}$ 657 (2005)

FURTHER READING Sherman, D. L. \& Brophy, P.J. Mechanisms of axon ensheathment and myelin growth. Nature Rev. Neurosci. 6, 683-690 (2005). WEB SITE

Brophy's laboratory: http://www.cnr.ed.ac.uk/ links/pbrophy.htm 